Research Article

Aurora Angela Pisano* and Paolo Fuschi

\title{
Stress evaluation in displacement-based 2D nonlocal finite element method
}

https://doi.org/10.1515/cls-2018-0010

Received Apr 06, 2018; accepted Apr 09, 2018

\begin{abstract}
The evaluation of the stress field within a nonlocal version of the displacement-based finite element method is addressed. With the aid of two numerical examples it is shown as some spurious oscillations of the computed nonlocal stresses arise at sections (or zones) of macroscopic inhomogeneity of the examined structures. It is also shown how the above drawback, which renders the stress numerical solution unreliable, can be viewed as the so-called locking in FEM, a subject debated in the early seventies. It is proved that a well known remedy for locking, i.e. the reduced integration technique, can be successfully applied also in the nonlocal elasticity context.
\end{abstract}

Keywords: Nonlocal displacement-based finite element method; 8-nodes Serendipity quadrilaterals; Nonlocal stress locking; Reduced integration technique

\section{Premises and motivations}

Nonlocal approaches in the field of continuum mechanics are nowadays widely used in a number of engineering applications (see e.g. [1-3]). The common feature of such approaches relies on the need to describe at a macroscopic level phenomena arising at a microscopic level, i.e. within the microstructure of the constituent material, that, indeed, play a significant role in the right description of the material/structural behavior. Typical examples of such circumstance can be traced back to the early seventies when dealing with the description of the stresses at a tip of a crack (see e.g. [4]) till more recent problems facing the analysis of structural elements made of materials containing nano-particles [5]. Wave dispersion, strain softening,

\footnotetext{
*Corresponding Author: Aurora Angela Pisano: Department PAU - via Melissari, I-89124 Reggio Calabria, Italy, University Mediterranea of Reggio Calabria; Email: aurora.pisano@unirc.it Paolo Fuschi: Department PAU - via Melissari, I-89124 Reggio Calabria, Italy, University Mediterranea of Reggio Calabria
}

concomitant size effects are, among others, well known examples (see e.g. [6-8] and references therein).

It is also well known that classical continuum mechanics fails in describing the above problems for the absence in the constitutive laws of any information coming from the microstructure. Indeed, the above referred nonlocal continuum approaches succeed in this challenge by introducing in the classical modeling an internal length material scale while keeping the hypothesis of continuity. There are different constitutive hypotheses that give rise to different nonlocal continuum theories and an huge amount of approaches and related numerical techniques proposed to face real problems (see e.g. [6] and [2] just to quote two milestone works). The list of quotable contributions is very long and out of the scope of the present paper. The nonlocal approach hereafter referred is the one known as nonlocal integral approach of Eringen and coworkers (see e.g. $[9,10]$ ). It is applied within the elasticity field and in a shape proposed by the authors in $[5,11-$ 14] and there named strain-difference-based nonlocal integral elasticity model. The stress is expressed as the sum of two contributions: a local standard one and a nonlocal one given in integral form and in terms of an averaged strain difference field. A nonlocal version of the finite element method, named NL-FEM, was also proposed in the above quoted papers on the base of a nonlocal total potential energy functional whose optimality conditions yield the governing equations of the pertinent boundary-valueproblem governed, besides the nonlocal stress-strain law, by standard equilibrium and compatibility conditions. The details of such formulation are given in the quoted paper and will be only briefly summarized hereafter. The main goal of the present paper is however strictly related to the already implemented NL-FEM and, as declared in the title, it concerns the stress evaluation within a displacementbased nonlocal formulation of such method.

The right evaluation of (nonlocal) stresses is, with no doubts, of utmost importance within a nonlocal numerical formulation. Stress based criteria to face fracture mechanics problems might be consistently rephrased for example, or nonlocal limit analysis for structural elements made of nonlocal materials, such as metal matrix nanocomposites, might be fields of possible fruitful application of the NL- 
FEM, but, obviously, many other nonlocal elastic problems may gain advantage from, or cannot renounce to, a correct description of the stress field. To the authors knowledge however this point has not been sufficiently addressed in the relevant literature and even in their previous works. Very often, the reliability and effectiveness of a nonlocal numerical approach is verified only by plotting the (nonlocal) strains. In many numerical applications, also arising from different nonlocal theories and hypotheses, the stress distribution is simply not given.

The cited NL-FEM was implemented by the authors with reference to plane stress cases and for 2D 8-node Serendipity displacement-based finite elements (FEs). When applied to benchmark problems in which nonlocality plays a prominent role such elements show indeed reliable results in terms of strain distributions but they definitively fail in stress description. Precisely, spurious oscillations come out making the solution in terms of stress completely unreliable. To face this problem, a numerical technique well known in the realm of standard FEM is proposed, namely the reduced integration procedure. The latter, first conceived by Zienkiewicz in the early seventies [15] see also [16-18] is, as known, one remedy among others, to treat the so-called locking in FEM. A sort of nonlocal locking can in facts be envisaged sharing many common features with the classical locking in FEM for a long debated, see e.g. $[19,20]$ and references therein. On taking into account that we are dealing with second order quadrilateral (nonlocal) elements and avoiding the circumstance of incompressible material, shear-locking and volumetric locking have to be excluded. The spurious stress oscillations observed at zones with (nonlocal) stress concentration rather resemble the so-called stress-locking exhibited by FEs with embedded discontinuities, see [21, 22] and references therein.

Two cases study are analyzed in Section 4 showing how an old numerical technique can be successfully applied in a novel version of the FEM. Some other considerations will be carried out in the same section concerning the possible source of stress-nonlocal-locking related to the analytical formulation of the method. The latter is briefly summarized in the next two sections. Concluding remarks point out the reached results drawing possible future steps of the ongoing research this paper belongs to.

Notation: Bold-face letters are used for vectors and tensors. The "dot" and "colon" products between vectors and tensors denote simple and double index contraction operations, respectively. For instance: $\boldsymbol{u} \cdot \boldsymbol{v}=u_{i} v_{i}, \boldsymbol{\sigma}: \boldsymbol{\varepsilon}=$ $\sigma_{i j} \varepsilon_{i j}, \boldsymbol{\sigma}: \boldsymbol{n}=\sigma_{i j} n_{i j}, \boldsymbol{D}: \boldsymbol{\varepsilon}=D_{i j h k} \varepsilon_{h k}$. The symbol $:=$ means equality by definition. In Section 3 , dealing with NL-FEM, a standard matrix notation is also used. Other symbols will be defined in the text at their first appearance.

\section{The nonlocal integral model}

The constitutive assumption which characterizes the adopted model is (see [12] for details):

$$
\begin{aligned}
& \boldsymbol{\sigma}(\boldsymbol{x})=\boldsymbol{D}(\boldsymbol{x}): \boldsymbol{\varepsilon}(\boldsymbol{x})-\alpha \int_{V} \mathcal{J}\left(\boldsymbol{x}, \boldsymbol{x}^{\prime}\right):\left[\varepsilon\left(\boldsymbol{x}^{\prime}\right)-\boldsymbol{\varepsilon}(\boldsymbol{x})\right] \mathrm{d} \boldsymbol{V}^{\prime} \\
& \forall\left(\boldsymbol{x}, \boldsymbol{x}^{\prime}\right) \in V
\end{aligned}
$$

where: $\boldsymbol{\sigma}(\boldsymbol{x})$ and $\boldsymbol{\varepsilon}(\boldsymbol{x})$ denote elastic stress and strain second order tensors, respectively; $\boldsymbol{D}(\boldsymbol{x})$ is the fourth order tensor of the elastic moduli (assumed variable in space to deal with macroscopic inhomogeneities of the material); $\mathcal{J}\left(\boldsymbol{x}, \boldsymbol{x}^{\prime}\right)$ is a nonlocal operator defined next; $\alpha$ is a (nonlocal) material parameter to be fixed on the base of experimental findings on the material in use. Looking at the r.h.s. of Eq. (1), besides the standard local first term of the stress (pertaining to a local material phase), a convolution integral appears as second term of the stress (pertaining to a nonlocal material phase). The latter defines a nonlocal contribution to the stress $\boldsymbol{\sigma}(\boldsymbol{x})$ arising from the postulated nonlocal behavior of the material whose influence is governed by the fixed material parameter $\alpha$. The nonlocal operator $\mathcal{J}\left(\boldsymbol{x}, \boldsymbol{x}^{\prime}\right)$ has the following shape:

$$
\begin{aligned}
& \mathcal{J}\left(\boldsymbol{x}, \boldsymbol{x}^{\prime}\right):=\left[\gamma(\boldsymbol{x}) \boldsymbol{D}(\boldsymbol{x})+\gamma\left(\boldsymbol{x}^{\prime}\right) \boldsymbol{D}\left(\boldsymbol{x}^{\prime}\right)\right] g\left(\boldsymbol{x}, \boldsymbol{x}^{\prime}\right)-\boldsymbol{k}\left(\boldsymbol{x}, \boldsymbol{x}^{\prime}\right) \\
& \forall\left(\boldsymbol{x}, \boldsymbol{x}^{\prime}\right) \in V
\end{aligned}
$$

with:

$$
\begin{gathered}
\gamma(\boldsymbol{x}):=\int_{V} g\left(\boldsymbol{x}, \boldsymbol{x}^{\prime}\right) \mathrm{d} V^{\prime} ; \\
g\left(\boldsymbol{x}, \boldsymbol{x}^{\prime}\right):=\lambda \exp \left(-\left|\boldsymbol{x}-\boldsymbol{x}^{\prime}\right| / \ell\right) ; \\
\boldsymbol{k}\left(\boldsymbol{x}, \boldsymbol{x}^{\prime}\right):=\int_{V} g(\boldsymbol{x}, \boldsymbol{z}) g\left(\boldsymbol{x}^{\prime}, \boldsymbol{z}\right) \boldsymbol{D}(\boldsymbol{z}) \mathrm{d} V^{z} .
\end{gathered}
$$

By inspection of Eq.(1) and positions (2-3), all derived by a thermodynamically consistent formulation given in [11], it appears how the strain-difference field at points $\left(\boldsymbol{x}, \boldsymbol{x}^{\prime}\right)$, namely $\left[\boldsymbol{\varepsilon}\left(\boldsymbol{x}^{\prime}\right)-\boldsymbol{\varepsilon}(\boldsymbol{x})\right]$ is eventually weighted by the function $g\left(\boldsymbol{x}, \boldsymbol{x}^{\prime}\right)$, known as attenuation function, through the operators $\gamma(\boldsymbol{x}), \boldsymbol{k}\left(\boldsymbol{x}, \boldsymbol{x}^{\prime}\right)$ and $\mathcal{J}\left(\boldsymbol{x}, \boldsymbol{x}^{\prime}\right)$ depending on it. Function $g\left(\boldsymbol{x}, \boldsymbol{x}^{\prime}\right)$, here assumed as bi-exponential with $\lambda=1 / 2 \pi \ell^{2}$ given by the normalization condition $\int_{V_{\infty}} g\left(\boldsymbol{x}, \boldsymbol{x}^{\prime}\right) \mathrm{d} V^{\prime}=1\left(V_{\infty}\right.$ being the infinite $3 D$ Euclidean 
space in which $V$ is embedded), introduces a second nonlocal material parameter of this adopted model. Such parameter, namely the internal length material scale $\ell$ depends on the microstructure's features, e.g. on the size and spacing of major inhomogeneities at small scale. The choice of the analytical shape of $g\left(\boldsymbol{x}, \boldsymbol{x}^{\prime}\right)$ and of the numerical value of $\ell$ concerns the connection between the (macroscopic) nonlocal integral continuum theory and the real nonlocal material behavior at atomistic (small) scale. The attenuation function decreases with increasing Euclidean distance between point $\boldsymbol{x}$ and $\boldsymbol{x}^{\prime}$ in $V$ vanishing beyond the so-called interaction radius or influence distance, say $L_{R}$. The nonlocal strain at a given point $\boldsymbol{x}$ is affected only by points $\boldsymbol{x}^{\prime}$ closer than $L_{R}$. In the limit of a local behavior, i.e. for $\ell \rightarrow 0, g\left(\boldsymbol{x}, \boldsymbol{x}^{\prime}\right)$ turns into a Dirac delta. The ability of $g\left(\boldsymbol{x}, \boldsymbol{x}^{\prime}\right)$ in capturing nonlocal phenomena is often tested by atomistic simulations (see e.g. [23-25]).

\section{The 2D displacement-based nonlocal finite element method}

The governing equation of a boundary-value-problem considering a constituent material obeying Eq.(1) can be obtained as the optimality conditions of the following nonlocal total potential energy functional:

$$
\begin{aligned}
\Pi[\boldsymbol{u}(\boldsymbol{x})] & :=\frac{1}{2} \int_{V} \nabla \boldsymbol{u}(\boldsymbol{x}): \boldsymbol{D}(\boldsymbol{x}): \nabla \boldsymbol{u}(\boldsymbol{x}) \mathrm{d} V+ \\
& +\frac{\alpha}{2} \int_{V} \nabla \boldsymbol{u}(\boldsymbol{x}): \gamma^{2}(\boldsymbol{x}) \boldsymbol{D}(\boldsymbol{x}): \nabla \boldsymbol{u}(\boldsymbol{x}) \mathrm{d} V+ \\
& -\frac{\alpha}{2} \int_{V} \int_{V} \nabla \boldsymbol{u}(\boldsymbol{x}): \mathcal{J}\left(\boldsymbol{x}, \boldsymbol{x}^{\prime}\right): \nabla \boldsymbol{u}\left(\boldsymbol{x}^{\prime}\right) \mathrm{d} V^{\prime} \mathrm{d} V+ \\
& -\int_{V} \boldsymbol{b}(\boldsymbol{x}) \cdot \boldsymbol{u}(\boldsymbol{x}) \mathrm{d} V-\int_{S_{t}} \boldsymbol{t}(\boldsymbol{x}) \cdot \boldsymbol{u}(\boldsymbol{x}) \mathrm{d} S .
\end{aligned}
$$

In Eq. (4): $\boldsymbol{u}(\boldsymbol{x})$ is the unknown displacement field in $V$; $\boldsymbol{b}(\boldsymbol{x})$ are the body forces; $\boldsymbol{t}(\boldsymbol{x})$ are the surface tractions acting on $S_{t}=S-S_{u}$ where $S$ denotes the boundary surface of $V$ and $S_{u}$ the portion where $\boldsymbol{u}(\boldsymbol{x})$ satisfy given kinematic boundary conditions, say $\boldsymbol{u}(\boldsymbol{x})=\overline{\boldsymbol{u}}(\boldsymbol{x})$.

A displacement-based nonlocal FEM can be formulated starting from a discretized version of Eq. (4) easily obtainable once introduced a modeling of the displacement field in terms of nodal displacement of the, let's say $N_{e}$, finite elements in which the domain $V$ is subdivided. Precisely, denoting with $(\cdot)_{n}$ a quantity referring to the $n$th FE in the mesh (with $n=1,2, \ldots N_{e}$ ) and with $\boldsymbol{N}_{n}(\boldsymbol{x})$ and $\boldsymbol{B}_{n}(\boldsymbol{x})$ the matrices of the interpolation (shape) func- tions and their derivatives, respectively, the displacement FE model is expressed by:

$$
\begin{gathered}
\boldsymbol{u}(\boldsymbol{x})=\boldsymbol{N}_{n}(\boldsymbol{x}) \boldsymbol{d}_{n}, \\
\boldsymbol{\varepsilon}(\boldsymbol{x})=\boldsymbol{B}_{n}(\boldsymbol{x}) \boldsymbol{d}_{n} \quad \forall\left(\boldsymbol{x}, \boldsymbol{x}^{\prime}\right) \in V_{n},
\end{gathered}
$$

where $\boldsymbol{d}_{n}$ is the vector collecting the nodal displacements of the element \#n occupying the volume $V_{n}$. Substituting $5(a, b)$ in (4) and rearranging, the discretized version of Eq. (4) can be given the shape:

$$
\begin{aligned}
\Pi\left[\boldsymbol{d}_{n}\right] & =\frac{1}{2} \sum_{n=1}^{N_{e}} \boldsymbol{d}_{n}^{T} \boldsymbol{k}_{n}^{\text {loc }} \boldsymbol{d}_{n}+\frac{\alpha}{2} \sum_{n=1}^{N_{e}} \boldsymbol{d}_{n}^{T} \boldsymbol{k}_{n}^{\text {nonloc }} \boldsymbol{d}_{n}+ \\
& -\frac{\alpha}{2} \sum_{n=1}^{N_{e}} \sum_{m=1}^{N_{e}} \boldsymbol{d}_{n}^{T} \boldsymbol{k}_{n m}^{\text {nonloc }} \boldsymbol{d}_{m}-\sum_{n=1}^{N_{e}} \boldsymbol{d}_{n}^{T} \boldsymbol{f}_{n} .
\end{aligned}
$$

In equation (6) the following positions have been made:

$$
\begin{gathered}
\boldsymbol{k}_{n}^{\text {loc }}:=\int_{V_{n}} \boldsymbol{B}_{n}^{T}(\boldsymbol{x}) \boldsymbol{D}(\boldsymbol{x}) \boldsymbol{B}_{n}(\boldsymbol{x}) \mathrm{d} V_{n}, \\
\boldsymbol{f}_{n}:=\int_{V_{n}} \boldsymbol{N}_{n}^{T}(\boldsymbol{x}) \boldsymbol{b}(\boldsymbol{x}) \mathrm{d} V_{n}+\int_{S_{t(n)}} \boldsymbol{N}_{n}^{T}(\boldsymbol{x}) \boldsymbol{t}(\boldsymbol{x}) \mathrm{d} S_{n},
\end{gathered}
$$

defining, as usual, the standard (local) element stiffness matrix and equivalent nodal force vector, respectively;

$$
\begin{gathered}
\boldsymbol{k}_{n}^{\text {nonloc }}:=\int_{V_{n}} \boldsymbol{B}_{n}^{T}(\boldsymbol{x}) \gamma^{2}(\boldsymbol{x}) \boldsymbol{D}(\boldsymbol{x}) \boldsymbol{B}_{n}(\boldsymbol{x}) \mathrm{d} V_{n}, \\
\boldsymbol{k}_{n m}^{\text {nonloc }}:=\int_{V_{n}} \int_{V_{m}} \boldsymbol{B}_{n}^{T}(\boldsymbol{x}) \mathcal{J}\left(\boldsymbol{x}, \boldsymbol{x}^{\prime}\right) \boldsymbol{B}_{m}\left(\boldsymbol{x}^{\prime}\right) \mathrm{d} V_{m} \mathrm{~d} V_{n},
\end{gathered}
$$

defining the nonlocal element stiffness matrices.

Remembering the definitions of the nonlocal operators $\gamma(\boldsymbol{x})$ and $\mathcal{J}\left(\boldsymbol{x}, \boldsymbol{x}^{\prime}\right)$, given by Eqs. (3a) and (2) respectively, as well as the property of the attenuation function $g\left(\boldsymbol{x}, \boldsymbol{x}^{\prime}\right)$, here assumed in the shape of Eq. (3b) that vanishes beyond the interaction radius $L_{R}$, the following can be stated. The nonlocal matrices $(8 \mathrm{a}, \mathrm{b})$ capture the nonlocal effects exerted on the current element $\# n$ by its neighbor elements, i.e. the ones falling within an influence zone of radius $L_{R}$ and centered on \#n, whose number, say $M_{e} \ll$ $N_{e}$, depends on $L_{R}$ and on the elements' size. In a FE context it is understood that: $\boldsymbol{x}$ is the position vector of the current Gauss point (GP) of the current element $\# n$ and $\boldsymbol{x}^{\prime}$ is the position vector of the current GP of the current neighbor element of \#n, say \#m with $m=1,2, \ldots M_{e}$; the influence zone (a circle of radius $L_{R}$ in 2D) is the centered at each GP of element \#n. 
Skipping all the details of the NL-FEM implementation given in [12], that can be referred for a deeper comprehension, it is worth noting that the assembling of the elements matrices $\boldsymbol{k}_{n}^{\text {loc }}, \boldsymbol{k}_{n}^{\text {nonloc }}$ and $\boldsymbol{k}_{n m}^{\text {nonloc }}$, the latter being a set of nonlocal matrices of element \# $n$ (a self-stiffness matrix, obtained for $m=n$, plus all the cross stiffness matrices obtained for $m=1,2, \ldots M_{e}$ with $m \neq n$ ) yields a nonlocal global matrix (which can be proved to be positive definite and symmetric) whose formal expression is:

$$
\begin{aligned}
\widehat{\boldsymbol{K}} & =\sum_{n=1}^{N_{e}} \boldsymbol{C}_{n}^{T} \boldsymbol{k}_{n}^{\text {loc }} \boldsymbol{C}_{n} \\
& +\alpha \sum_{n=1}^{N_{e}}\left[\boldsymbol{C}_{n}^{T} \boldsymbol{k}_{n}^{\text {nonloc }} \boldsymbol{C}_{n}-\sum_{m=1}^{M_{e}} \boldsymbol{C}_{n}^{T} \boldsymbol{k}_{n m}^{\text {nonloc }} \boldsymbol{C}_{m}\right] .
\end{aligned}
$$

In equation (9) $\boldsymbol{C}_{n}$ and $\boldsymbol{C}_{m}$ are the connectivity matrices enlarging the element matrices to global dimensions and the sums of such enlarged elements' matrices mimic the assembling procedure. It is clear that the nonlocal global matrix $\widehat{\boldsymbol{K}}$ is more populated than in the standard local FEM due to the presence of the elements' cross-stiffness matrices $\boldsymbol{k}_{n m}^{\text {nonloc }}$.

\subsection{Stress evaluation}

As said, the NL-FEM was already numerically implemented by the authors, precisely a 2D formulation for plane stress problems has been developed and successfully applied to quite a few examples and benchmark problems ([12, 13]).

The implemented NL-FEs are isoparametric 8-nodes Serendipity quadrilaterals. This choice was suggested by a number of reasons well known in the standard (local) FEM formulation, that is: $i$ ) second-order elements outperform first-order ones in problems with stress concentrations being also ideally suited for the analysis of (stationary) cracks, one of the future goal of the present promoted NL-FEM; ii) second-order elements capture geometric features, such as curved edges, with fewer elements than firstorder ones providing also high accuracy in stress concentration regions; iii) they do not suffer shear locking when model bending; $i v$ ) over all, their isoparametric formulation represents the biggest breakthrough in the implementation of the FEM for its capability to model problems (structures) geometries of any shape and size by working on mapping.

All the numerical integrations have been carried on by Gauss quadrature integration rule with a full integration, i.e. taking into account $3 \times 3 \mathrm{GPs}$ per element. It is worth noting that for second-order elements Gauss integration is the more used (at least in the local context) because it is efficient and it is especially suited to the polynomial product interpolation adopted in these elements, a quality serviceable also in the present nonlocal context.

The global (nonlocal) equation system was solved by a standard and robust commercial package, employing the RTR Cholesky factorization for symmetric positive definite matrices with an iterative refinement on the solution vector to improve the accuracy, getting the (nonlocal) nodal displacements and the strains at the GPs of the NL-FEs.

As shown by the numerical findings reported in the quoted papers of the authors and as briefly illustrated in the next section, the (nonlocal) strains appear very good: they are not affected by mesh dependence, they are able to capture size-effects and, even if not compared with laboratory findings on real prototypes practically absent in the relevant literature, they look very sensible and reliable showing also the right sensitivity to the parametric analyses obtained by varying the fixed values of the nonlocal material parameters $\alpha$ and $\ell$ also in presence of macroscopic inhomogeneities.

Nevertheless, when such solutions (in terms of nonlocal strains) were used back in a discretized FE form of Eq. (1) to get the stresses at the GPs of the NL-FEs, such stresses result to be affected by spurious oscillations. As observed in Section 1, avoiding the case of incompressibility which might produce volumetric locking, a sort of nonlocal-stress-locking can be envisaged. Precisely, spurious stress oscillations arise at zones with macroscopic inhomogeneities spreading all over the adjacent zones rendering the stress solution completely unreliable. The proposed remedy to such drawback is ancient and is a numerical remedy, namely the reduced integration proposed by Zienkiewicz in [15-17], see also [18-20] for a deeper comprehension. It is worth reminding that full integration means that the minimum integration order required for an exact integration of the strain energy for an undistorted element with linear material properties is used. The reduced integration is then the integration rule that is one order less than the full integration one.

In the 8-nodes isoparametric NL-FEs here adopted reduced integration means an integration performed on $2 \times 2$ GPs per element. As shown in the next section such reduced integration does not affect the response in terms of (nonlocal) strains that is it does not influence the computed displacements and therefore most likely it does not affect the evaluations on the terms of the nonlocal global matrix $\widehat{\boldsymbol{K}}$. Indeed, the reduced integration improves dramatically the response in terms of stresses computed at the GPs of the NL-FEs yielding, for example, the "exact solution" in terms of stresses for a benchmark problem ana- 
lyzed in next section and characterized by a known constant stress on the whole BVP domain.

By investigating the trend of the single stress contributions at the r.h.s. of Eq. (1), taking into account Eqs. (2, 3) and obtained by full and reduced integration, it has been noted that the "source of stress oscillations" is the integral over the whole domain $V$ of the nonlocal operator $\mathcal{J}\left(\boldsymbol{x}, \boldsymbol{x}^{\prime}\right)$. The reduced integration definitely overcome this drawback. It is worth noting that the operator $\mathcal{J}\left(\boldsymbol{x}, \boldsymbol{x}^{\prime}\right)$ is also integrated when computing the cross-stiffness element matrices $\boldsymbol{k}_{n m}^{n o n l o c}$, see Eq. (8b), but in this case the integral is carried on or, let's say, is confined to, the subdomains (volumes) $V_{n}$ and $V_{m}$ of the involved elements, a circumstance that, as numerically detected, does not produce any form of instability.

The idea of speaking of nonlocal-stress-locking relies firstly on the nature of the observed stress oscillations (obtained by full integration) for which the term to describe them seems appropriate but, secondly, it relies on the observation that it resembles, at least in many formal aspects, the so-called stress-locking exhibited by (local) FEs with embedded discontinuities, see e.g. [21, 22] and references therein. Such circumstance has however to be further investigated being just an hint for a better needful understanding of the observed phenomenon.

Concerning the adoption of the reduced integration in the present nonlocal elastic context the following further general remarks can be made.

Remark 1. A FE displacement-based model, also in the present nonlocal form, is inexact and usually it errs by being too stiff. Borrowing from [18], this is a congenital error of any displacement-based formulation where the displacements are "on the whole" underestimated and the stiffness of the model is "on the whole" overestimated as a results of the "internal displacement constrains" implicitly imposed on the solution by the assumed displacement model. If the error made with a reduced numerical integration compensates for the overestimation of structural stiffness due to finite element discretization improved results may be expected.

Remark 2. Reduced integration leads (in the local context) to rank deficiency of the stiffness matrix and "spurious" zero energy modes or "hour-glass" modes. This drawback seems here mitigated by a stiffness matrix which for each NL-FE collects contributions coming from the neighbors elements. Moreover, the hour-glass modes, characterized by strains not detected at the (reduced) integration points so furnishing nonphysical response modes with deformations but no strains (zero-energy modes), reduce as known to only one mode in the second-order Serendipity 8-nodes quadrilaterals and it does not propagate to neighbors elements so it will not occur in a mesh with more than one element as it happens in the local FEM.

Remark 3. On taking into account what observed above on the integration of the nonlocal operator $\mathcal{J}\left(\boldsymbol{x}, \boldsymbol{x}^{\prime}\right)$, which is integrated on the whole $V$ in Eq. (1) being responsible of the stress oscillations and integrated only on the subdomains $V_{n}$ and $V_{m}$ when computing the cross-stiffness matrices of Eq. (8b), a selective reduced integration might be also envisaged when dealing with more complex nonlocal problems in which reduced integration could be used only for the stress evaluation by Eq. (1).

At closure of this section it is difficult, as in the standard FEM formulation, to provide a general guideline, applicable to all types of problems, on whether to use reduced or higher order integration. It is reasonable to assume, as in the standard FEM, that reduced integration is generally better suited for undistorted elements as the ones considered in the following examples, whereas higher order (or full) integration tends to work better as the element becomes more distorted. To this concern further investigations within the nonlocal context are the object of an ongoing research.

\section{Cases study}

\subsection{Bar-like structure under tension}

The nonlocal elastic nonhomogeneous bar-like structure of Figure 1 is analyzed first. This problem was solved in [11] following a 1D formulation, i.e. referring to the bar's axis (dashed line) in Figure 1 and assuming the bar, of unit cross-section, fixed at the end $x=0$ and subjected to a given displacement $\bar{u}_{x}=0.2 \mathrm{~cm}$ at the end $x=L=100 \mathrm{~cm}$. The bar is characterized by a macroscopic inhomogeneity being made, by hypothesis, of a material with a piecewise homogeneous Young modulus $E_{1}=84 \mathrm{GPa}$ and $E_{2}=210$ $\mathrm{GPa}$ in the first and in the second half, respectively. The Poisson ratio is $v=0.2$ on the whole bar. The nonlocal

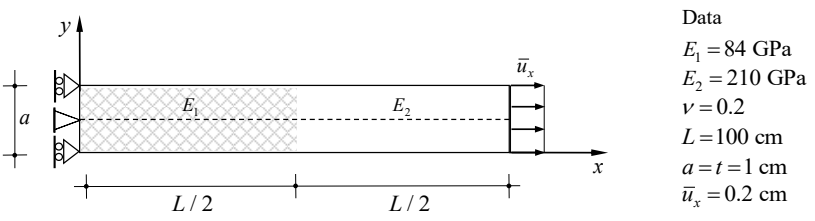

Figure 1: Bar-like structure in tension: geometry, loading, boundary conditions and material data. 

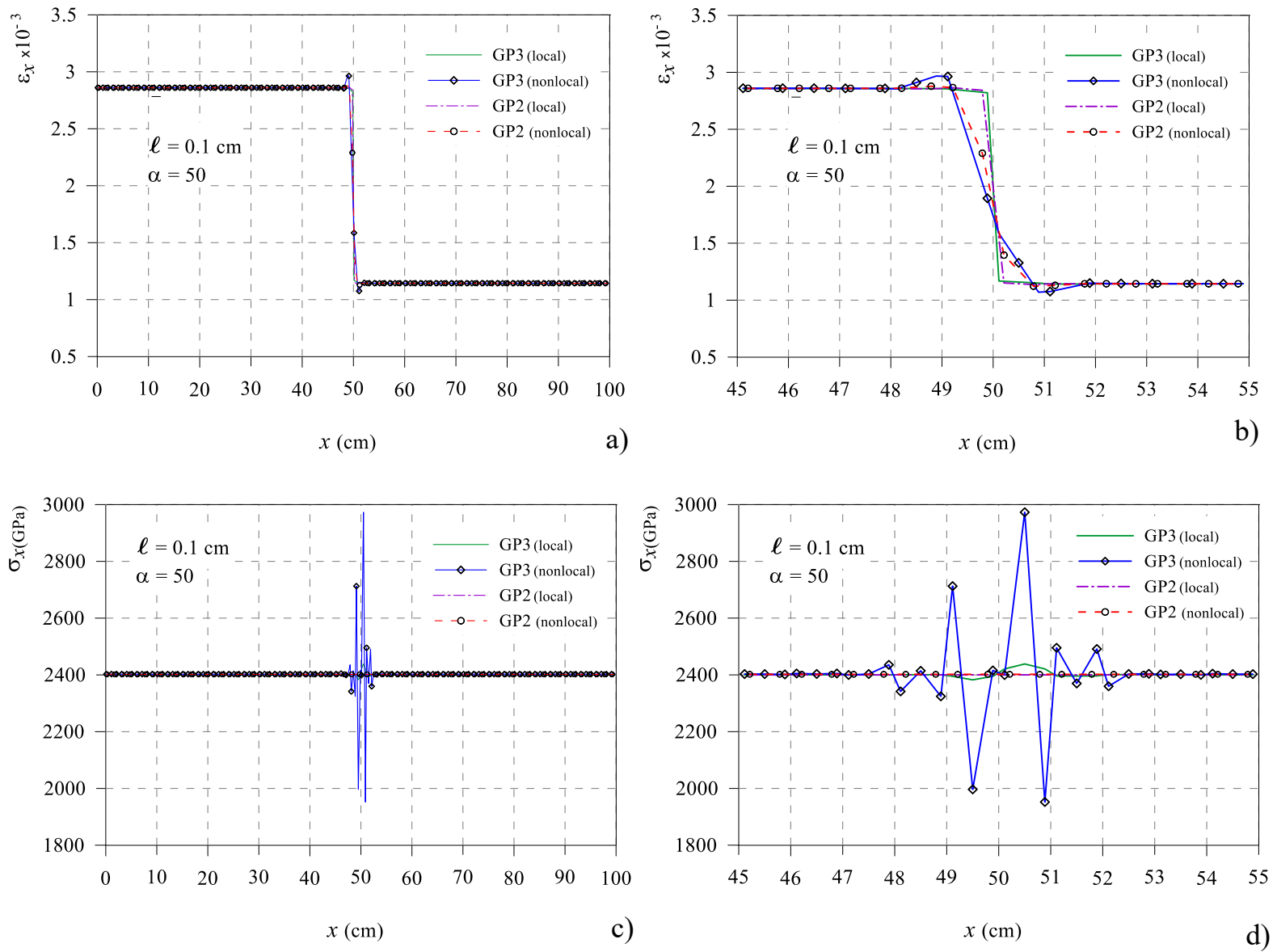

Figure 2: Local and nonlocal solution obtained with full (GP3 curves) and reduced (GP2 curves) integration for the bar of Figure 1 with internal length $\ell=0.1: a$ ) and $b$ ) strain $\varepsilon_{X}$ and zoom at the bar mid zone respectively; $c$ ) and $d$ ) stress $\sigma_{x}$ and zoom at the bar mid zone respectively.

material parameters $\alpha$ is assumed equal to 50 while three values $\ell=0.1,0.5,1(\mathrm{~cm})$ of the internal length will be assumed next.

The solution procedure in 1D is quite simple because of the stress $\sigma_{x}$ being constant in the whole bar for equilibrium. Starting from a 1D version of the constitutive model (1) it is easy to get a governing Fredholm integral equation of the second kind which can be uniquely solved, for instance by a successive iteration procedure, applying eventually the bar boundary conditions. The analytical 1D solution in terms of strains and stresses, obtained following the rationale given in [11] and not reported for brevity, has been considered as benchmark for the here followed 2D NL-FEM treatment of the same problem. To this concern in the 2D simulation (refer again to Figure 1) apposite constrains have been applied on the fixed edge at $x=0$ that, together with a low value of the Poisson ratio, render the 2D bar-like structure as much as possible equivalent to its
1D version for which the alternative analytical solution is available.

The bar-like structure has been discretized with one row of 100 8-nodes NL-FEs Serendipity quadrilaterals and the obtained results are given in Figures 2-4) for three values of the internal length $\ell$ assumed in the numerical analyses.

Figures 2(a,b), 3(a,b) and 4(a,b) plot, along the bar's axis, the local and nonlocal strains $\varepsilon_{x}$ for $\ell=0.1,0.5$ and 1 respectively, all the Figures (b) give a zoom of the curves of Figures (a) around the midsection of the bar, i.e. where the Young modulus abruptly changes. By inspection of the strain curves it appears how, beyond the already known difference between the local and nonlocal strains, no significant mismatch exists, they are coincident in practice, between the strain curves (GP3 nonlocal and GP2 nonlocal) obtained by full and reduced integration. The analytical benchmark solution in terms of nonlocal strains, not 

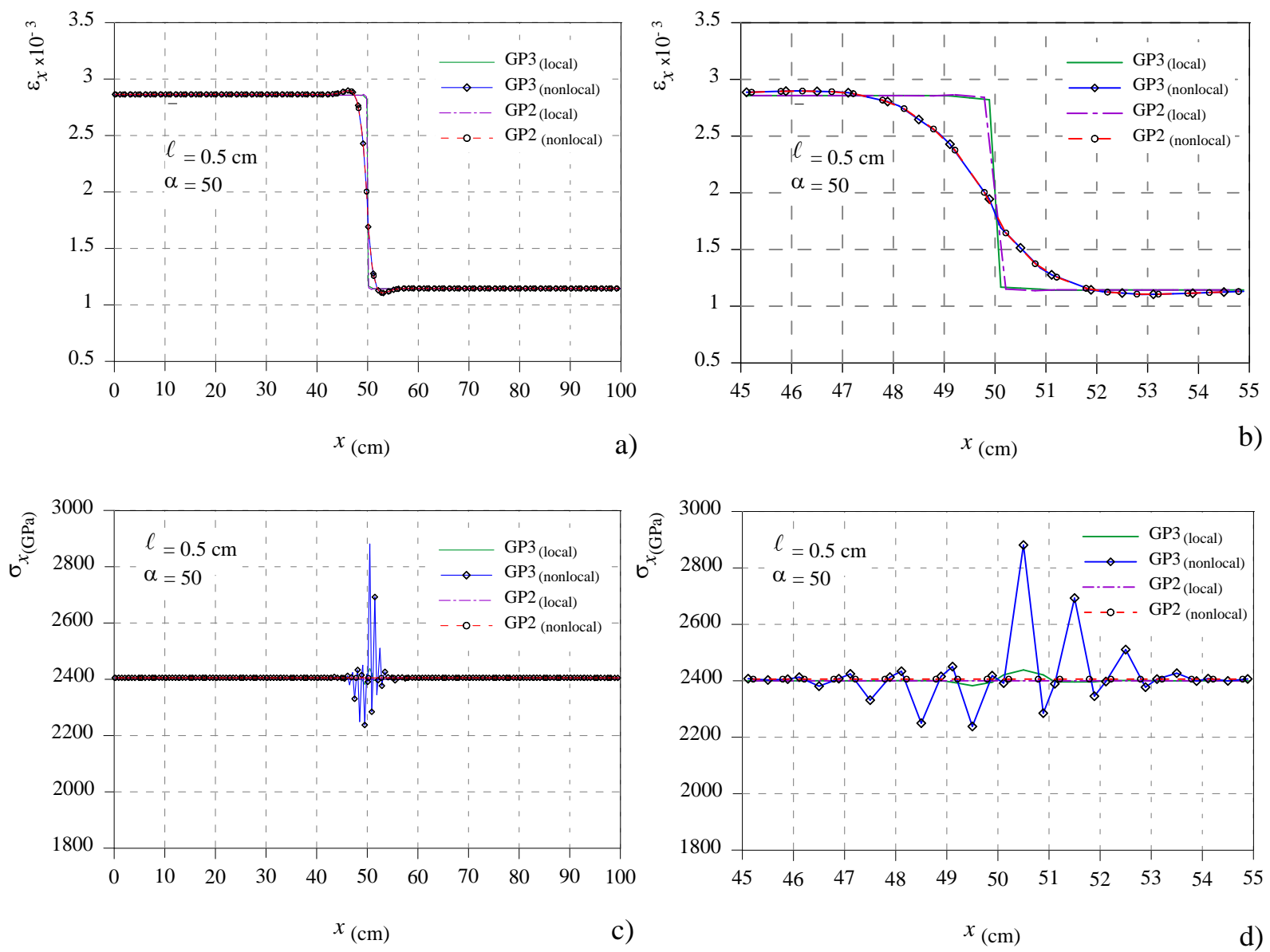

Figure 3: Local and nonlocal solution obtained with full (GP3 curves) and reduced (GP2 curves) integration for the bar of Figure 1 with internal length $\ell=0.5: a$ ) and $b$ ) strain $\varepsilon_{X}$ and zoom at the bar mid zone respectively; $c$ ) and $d$ ) stress $\sigma_{X}$ and zoom at the bar mid zone respectively.

potted for clarity, is also exactly the same of the curves obtained by 2D NL-FEM treatment.

On the contrary, looking at Figures 2(c,d), 3(c,d) and $4(\mathrm{c}, \mathrm{d})$ plotting the local and nonlocal stress $\sigma_{x}$ for $\ell=0.1,0.5$ and 1 respectively, where once again all the Figures (b) give a zoom of the stress curves around the midsection of the bar, the following can be stated. The previously discussed spurious nonlocal stress oscillations, here named nonlocal-stress-locking, arise at the bar midsection spreading around it when using full integration (GP3 curves). The nonlocal stresses given by reduced integration (GP2 curves) give conversely the right solution, i.e. a stress distribution constant over the whole bar by equilibrium and coincident with the local and analytical ones as it has to be. The discussed effectiveness of the reduced integration is so proved.

\subsection{Plate with inclusion}

The nonhomogeneous square plate of Figure 5 has been analyzed focusing the attention on the nonlocal stress evaluation.

The same example was treated in [12] giving the response only in terms of nonlocal strains and showing the sensitivity of the solution to the nonlocal material parameters $\alpha$ and $\ell$, the mesh independency, the ability of the NL-FEM in capturing size-effects.

The plate geometry, material data, loading and boundary conditions are also given in Figure 5. A macroscopic inhomogeneity is given by a squared inclusion at the plate's core with a Young modulus $E_{1}=84 \mathrm{GPa}$. In the remaining part of the plate a Young modulus $E_{2}=210 \mathrm{GPa}$ is assumed. The Poisson ratio is $v=0.2$ while the value $\alpha=50$ has been assumed in the NL-FE analysis performed with a mesh of 40x40 8-nodes, isoparametric, Serendipity quadrilaterals. The values $\ell=0.05,0.1,0.5(\mathrm{~cm})$ have 

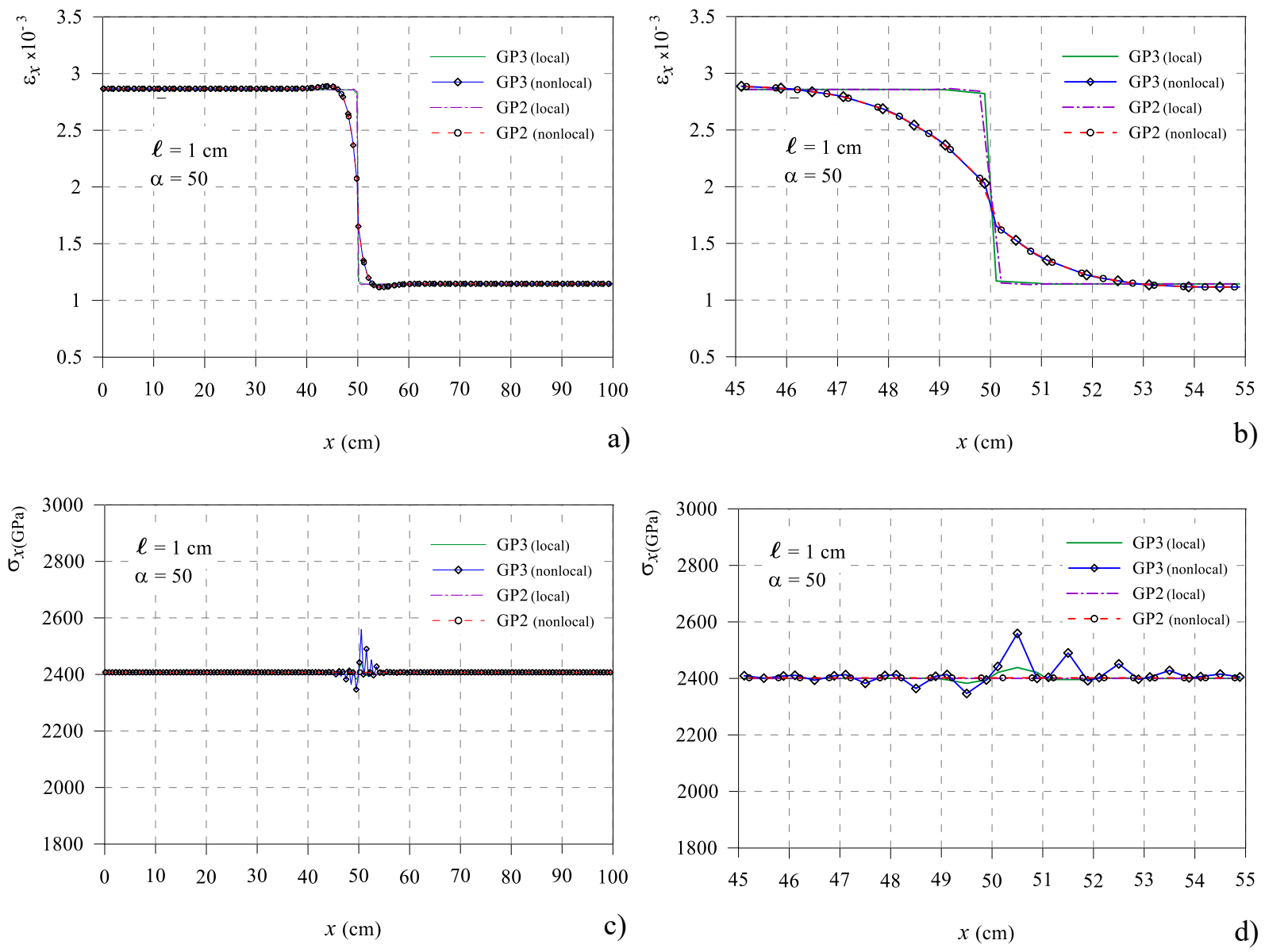

Figure 4: Local and nonlocal solution obtained with full (GP3 curves) and reduced (GP2 curves) integration for the bar of Figure 1 with internal length $\ell=1: a$ ) and $b$ ) strain $\varepsilon_{x}$ and zoom at the bar mid zone respectively; $c$ ) and $d$ ) stress $\sigma_{x}$ and zoom at the bar mid zone respectively.

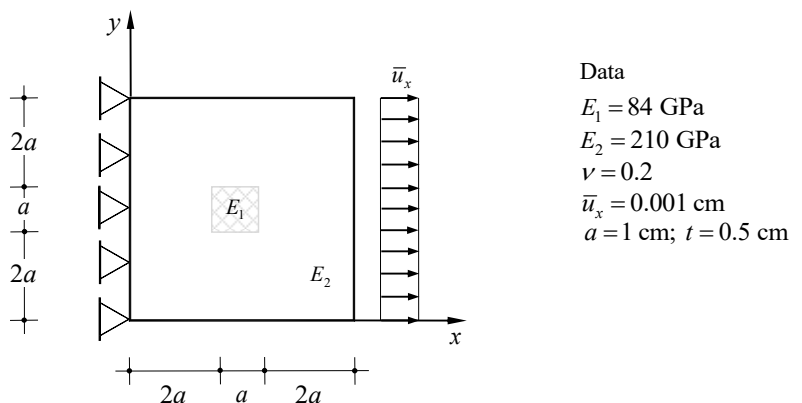

Figure 5: Plate with inclusion: geometry, loading, boundary conditions and material data.

been considered. The plate is fixed at edge $x=0$ and it is subjected to imposed uniformly distributed displacements $\bar{u}_{x}=0.001 \mathrm{~cm}$ at the edge $x=5 a$ (with $a=1 \mathrm{~cm}$ ).

Figures 6(a-c) show, along the mid side section of the plate at $y=2.5 a$, the nonlocal strains $\varepsilon_{x}$ against the local ones, both computed with full and reduced integration.
Beyond the known mismatch between the local and nonlocal strains $\varepsilon_{x}$, by inspection of the nonlocal strains curves, named GP3 nonlocal and GP2 nonlocal, it is confirmed also in this case that full or reduced integration does not affect the nonlocal solutions in terms of strains.

Figures $7(\mathrm{a}-\mathrm{c})$ plot the stress $\sigma_{x}$, i.e. the preeminent stress component along the loading direction, at the mid side section for $y=2.5 a$. Once again the stresses obtained by a full integration (GP3 nonlocal curves) show spurious oscillations arising at sections where the Young modulus changes. The latter spread within the surrounding areas rendering the nonlocal stress solution unreliable. The nonlocal stresses given by reduced integration (GP2 nonlocal curves) eliminate completely the undesirable oscillations.

It is worth noting that in both examples the "amplitude" of the spurious oscillations increases with decreasing values of the internal length material scale $\ell$. 

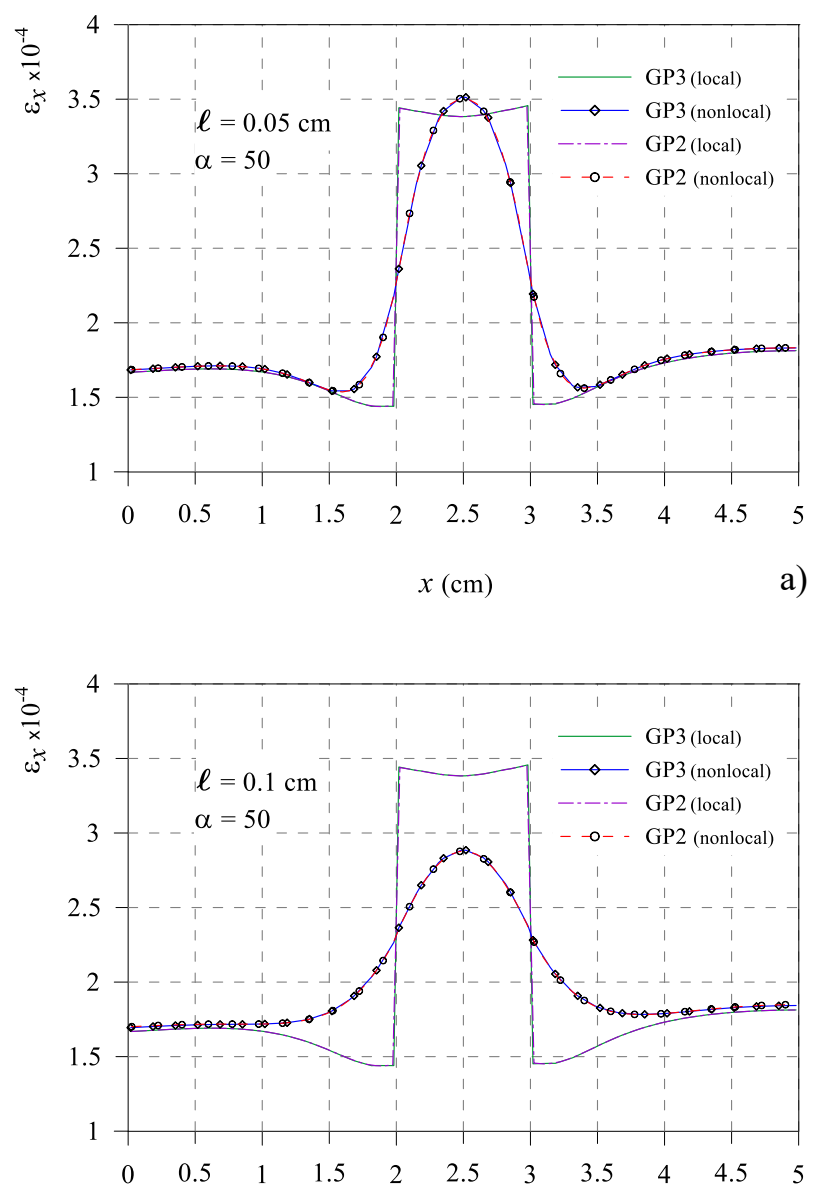

$x(\mathrm{~cm})$

b)

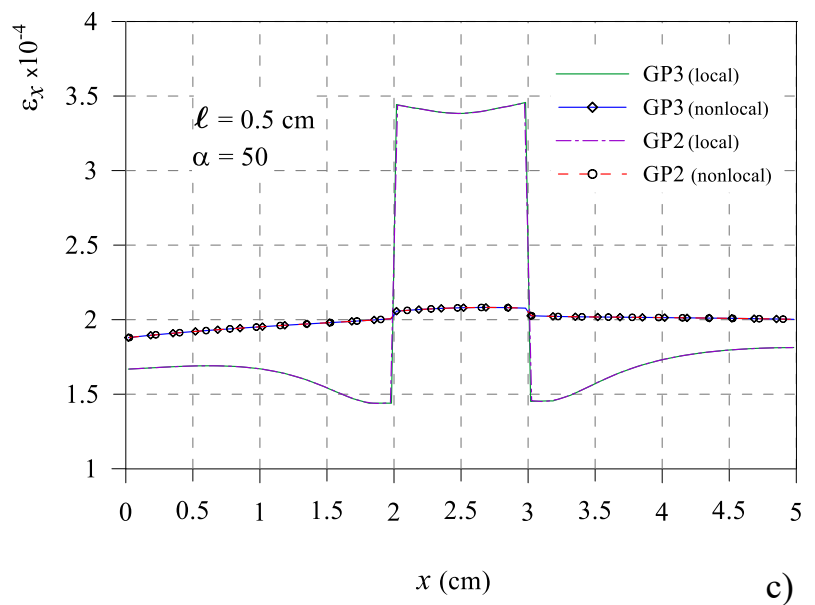

Figure 6: Local and nonlocal strain profiles $\varepsilon_{\chi}$, obtained with full (GP3 curves) and reduced (GP2 curves) integration for the plate of Figure 5 , at the mid section $\mathrm{y}=2.5 \mathrm{a}: a$ ) for $\ell=0.05 ; b$ ) for $\ell=0.1 ; c$ ) for $\ell=0.5$.
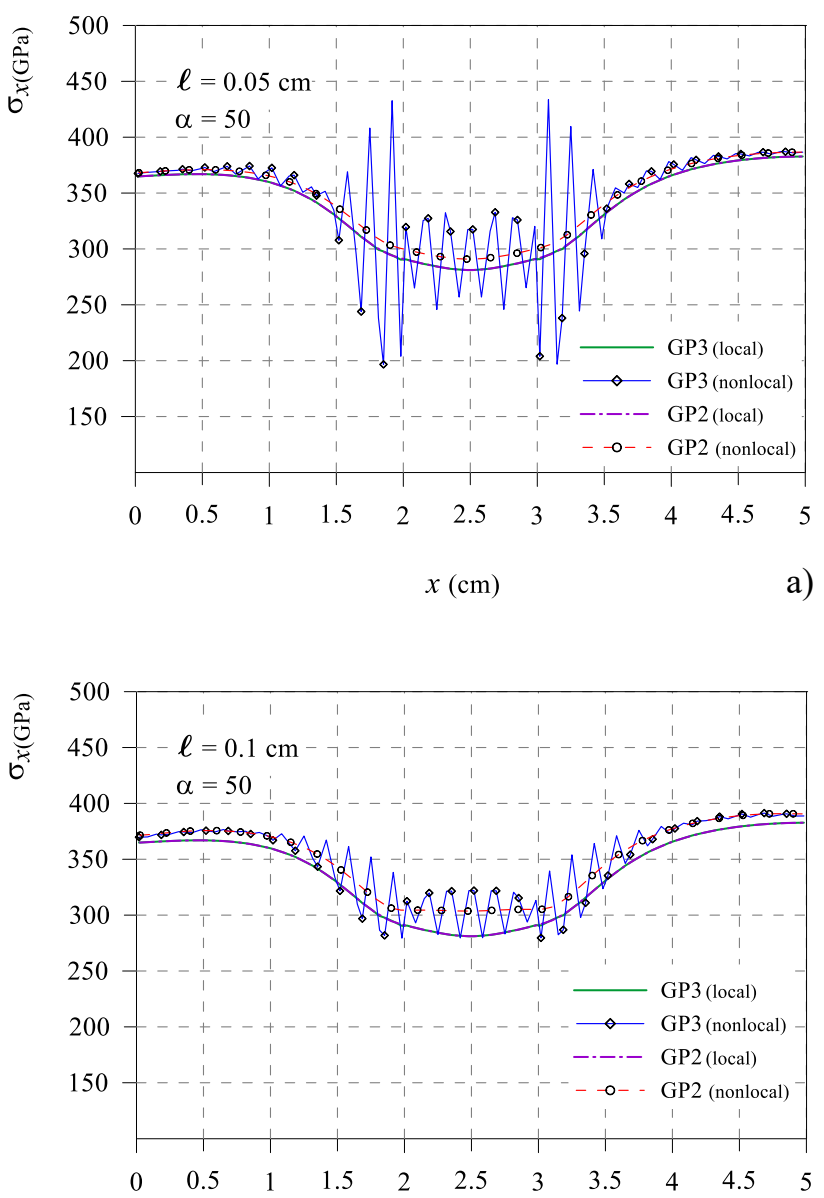

$x(\mathrm{~cm})$

b)

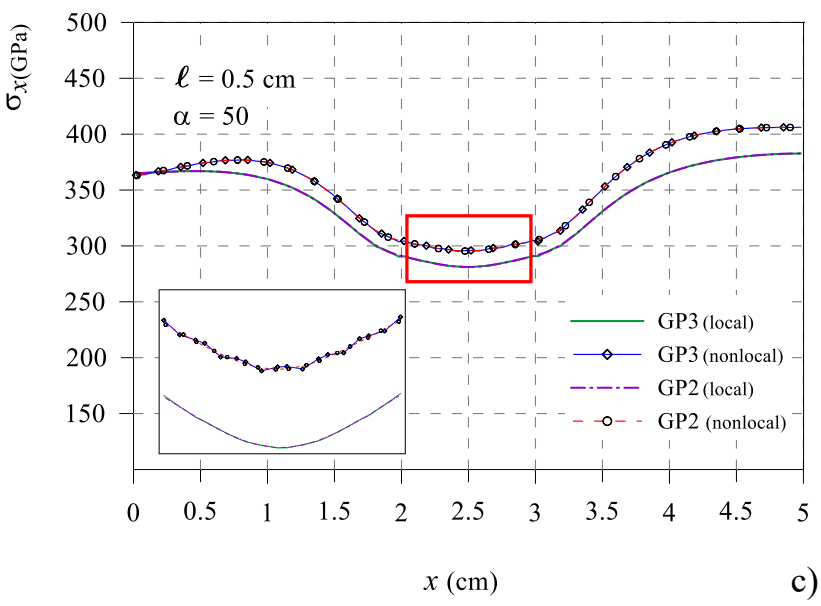

Figure 7: Local and nonlocal stress profiles $\sigma_{x}$, obtained with full (GP3 curves) and reduced (GP2 curves) integration for the plate of Figure 5 , at the mid section $\mathrm{y}=2.5 \mathrm{a}: a$ ) for $\ell=0.05 ; b$ ) for $\ell=0.1 ; c$ ) for $\ell=0.5$. 


\section{Concluding remarks}

The paper has very briefly summarized a nonlocal formulation of the displacement-based finite element method previously implemented by the authors with reference to a nonlocal elastic constitutive model of integral type also allowing to treat structures made of nonhomogeneous materials. Attention has then been focused on the goal of the work, i.e. the stress evaluation within the nonlocal FEM procedure.

A numerical drawback, characterized by the outset of spurious stress oscillations at sections (or zones) of macroscopic inhomogeneities of the examined nonlocal elastic structures here given by abrupt changes of the Young modulus, has been pointed out. A sort of nonlocal locking, named nonlocal-stress-locking, has been envisaged.

A well known numerical technique to avoid locking in FEM has been re-proposed in this context, i.e. the reduced integration. The numerical findings on the two tackled problems, one of which allowing an alternative analytical solution useful for comparison purposes, seem to validate the proposed remedy.

The topic, far to be exhaustively treated, has to be viewed as the authors' will of sharing what experienced in the numerical determination of the stresses in a nonlocal context treated by a displacement-based NL-FEM procedure. The proposed remedy opens the way to a wide range of problems as, for example, the stresses determination in the field of (nonlocal) elastic fracture mechanics with the consequent adoption of nonlocal stress-based criteria.

\section{References}

[1] A.C. Eringen. Nonlocal continuum field theories. SpringerVerlag New York (2002).

[2] Z.P. Bažant, L. Cedolin. Stability of structures: elastic, inelastic, fracture and damage theories. World Scientific Publishing Company Ed. (2010).

[3] M.A. Eltaher, M.E. Khater, Samir A. Emam. A review on nonlocal elastic models for bending, buckling, vibrations, and wave propagation of nanoscale beams. Applied Mathematical Modelling 40 (2016) 4109-4128.

[4] A.C. Eringen, B.S. Kim. Stress concentration at the tip of a crack. Mechanics Research Communications 1 (1974) 233-237.

[5] P. Fuschi, A.A. Pisano. Ultimate load prediction of MMNCs structures. Composites Part B 125 (2017) 175-180.

[6] Z.P. Bažant, M. Jirásek. Nonlocal integral formulations of plasticity and damage: survey of progress. Journal of Engineering Mechanics 11 (2002) 1119-1149.
[7] V.V. Zozulya. Micropolar curved rods. 2-D, high order, Timoshenko's and Euler-Bernoulli models. Curved and Layered Structures 4 (2017) 104-118.

[8] V.V. Zozulya. Nonlocal theory of curved rods. 2-D, high order, Timoshenko's and Euler-Bernoulli models. Curved and Layered Structures 4 (2017) 221-236.

[9] A.C. Eringen. On differential equations of nonlocal elasticity and solutions of screw dislocation and surface waves. J. Appl. Phys. 54(9) (1983) 4703-4710.

[10] A.C. Eringen. Theory of nonlocal elasticity and some applications. Res Mechanica 21 (1987) 313-342.

[11] C. Polizzotto, P. Fuschi, A.A. Pisano. A nonhomogeneous nonlocal elasticity model. European Journal of Mechanics A/Solids 25 (2006) 308-333.

[12] P. Fuschi, A.A. Pisano, D. De Domenico. Plane stress problems in nonlocal elasticity: finite element solutions with a straindifference-based formulation. Journal of Mathematical Analysis and Applications 431 (2015) 714-736.

[13] A.A. Pisano, P. Fuschi. Structural symmetry within nonlocal integral elasticity: theoretical issues and computational strategies. Curved and Layered Structures 4 (2017) 1-7.

[14] A.A. Pisano, P. Fuschi. Structural symmetry and boundary conditions for nonlocal symmetrical problems. Meccanica 53 (2018) 629-638.

[15] O.C. Zienkiewicz, R.L. Taylor, J.M. Too. Reduced integration technique in general analysis of plates and shells. International Journal Numerical Methods in Engineering 3 (1971) 275-290.

[16] O.C. Zienkiewicz, E. Hinton. Reduced integration, function smoothing and non-conformity in finite element analysis (with special reference to thick plates). J. Franklin Institute 302 (1976) 443-461.

[17] O.C. Zienkiewicz, R.L. Taylor. The finite element method. McGraw-Hill book company (1989).

[18] K-J. Bathe. Finite element procedures. New Jersey: Prentice-Hall (1996).

[19] T.J.R. Hughes. The finite element method, linear static and dynamic finite element analysis. New Jersey: Prentice-Hall (1987).

[20] D.S. Burnet. Finite element analysis. From concepts to applications. Addison-Wesley Publishing company (1987).

[21] M. Jirásek. Comparative study on finite element with embedded cracks. Computer Methods in Applied Mechanics and Engineering 188 (2000) 307-330.

[22] M. Jirásek, Th. Zimmermann. Embedded crack model: I Basic formulation, II Combination with smeared cracks. International Journal for Numerical Methods in Engineering 50 (2001) 12691290 and $1291-1305$.

[23] R.C. Picu. On the functional form of non-local elasticity kernels. Journal of the Mechanics and Physics of Solids 50(9) (2002) 1923-1939.

[24] V. Sundararaghavan, A.M. Waas. Non-local continuum modeling of carbon nanotubes: Physical interpretation of non-local kernels using atomistic simulations. Journal of the Mechanics and Physics of Solids 59(6) (2011) 1191-1203.

[25] S. Ghosh, A. Kumar, V. Sundararaghavan, A.M. Waas. Non-local modeling of epoxy using an atomistically-informed kernel. International Journal of Solids and Structures 50 (2013) 28372845. 